\title{
The Energy Consumption Forecasting in China Based on ARIMA Model
}

\author{
Junwei Miao \\ Department of Economic Management, North China Electric Power University, Baoding, 071003, \\ China
}

1506355739@qq.com

Keywords: energy consumption, ARIMA model; forecast, China

\begin{abstract}
China's energy consumption will continue to rise rapidly. It is necessary to forecast China's energy consumption to formulate energy and economic policy. This study establish a ARIMA model and forecast the energy consumption in future. The results show ARIMA model performs high prediction accuracy. It is suitable for energy consumption. It is also observed that China's energy consumption is likely to increase during 2012-2020. Energy consumption in 2020 is projected to $4450 \mathrm{Mtce}$ in 2020 , lower than target value. The results are helpful to policy formulation in China.
\end{abstract}

\section{Introduction}

Energy is essential for the functioning of all activities be it a developed or developing nation [1]. Over the past few decades, high growth rate of China's economy relies heavily on energy consumption. The energy consumption in China has increased from 594.47 million tons of standard coal (Mtce) in 1981 to 3617.32 Mtce in 2012 [2]. Rapid economic growth in China led to increasing energy consumption. To some extent, excessive energy consumption has surpass the limits in resource and environment carrying capacity. Energy shortage has become one of the main challenges of the 21st century in China. To control the growth rate and improve the efficiency of China's energy consumption has become a top priority of China's future economic development. Modeling and forecasting of the energy consumption is important for making energy and economic policies of a developing country like China.

\section{Discussed Problems}

Energy forecasting methods at home and abroad contains co-integration and error correction method, scenario analysis, input output method, time series analysis et al. The prediction method of time series analysis primarily analyzes variation trend of energy consumption and establish a time series mode. Hor C.L. predicts electricity consumption during 1998-2003 [3]. Liu Yong forecasts the energy consumption in China during 2000-2006 [4]. All Results suggest ARIMA has high precision, stable predictions and suitable for predicting energy consumption. Therefore, in this paper, ARIMA model is used to forecast China's energy consumption in future.

\section{The Solution Based On ARIMA Model}

Model Structure .ARIMA model. It is developed by Box and Jenkins in 1970. It is a time series analysis method based on the theory of random. The model has three basic types: Auto-regressive model (AR(P)), the moving average model (MA (q)) and auto-regressive moving average model (ARMA (p, q)) [6]. ARIMA model combines differential operation with ARMA model. The model structure is as followed [7]: 


$$
\left\{\begin{array}{c}
\nabla^{d} x_{t}=\mu+\frac{\Theta(B)}{\Phi(B)} C \\
E\left(\varepsilon_{t}\right)=0, \operatorname{Var}\left(\varepsilon_{t}\right)=\sigma_{\varepsilon}^{2}, E\left(\varepsilon_{t} \varepsilon_{s}=0\right), s \neq t \\
E\left(\varepsilon_{t} \varepsilon_{s}=0\right), \forall s<t
\end{array}\right.
$$

Where, $\mu$ is the mean value of sequences $\left\{x_{t}\right\}, B$ is delay operator; $\nabla^{d}=(1-B)^{d} ; \Phi(B)=1-\phi_{1} B-\cdots-\phi_{p} B^{p}$ is $p$-index regression coefficients polynomial; $\Theta(B)=1-\theta_{1} B-\cdots \theta_{q} B^{q}$ is $q$-index moving average coefficients polynomial; $\varepsilon_{t}$ is zero-mean white noise sequence.

\section{Method Steps.The ARIMA Model Mainly Includes Four Steps:}

(1) The stationary test for original sequence. The differential transform is employed to adjust original sequence to meet the stability in case that original sequence is not stationary.

(2) By calculating the the statistics that describe sequence characteristics (i.e.auto-correlation coefficient and partial auto-correlation coefficient), the parameters $p$ and $q$ in ARIMA model will be determinated.

(3) The estimation of the unknown parameters in the model and examination the rationality of this model.

(4) Diagnostic analysis to confirm that the obtained model is consistent with the observed data characteristics.

\section{Computational Examples And Analysis}

Data Source.In this paper, the data of energy consumption is collected from China Statistical Yearbook [5]. The total energy consumption in China was a significantly upward trend (Fig.1). The total energy consumption in China has increased from 1455 Mtce in 2000 to 3610.83 Mtce in 2012. The growth rate of energy consumption is relatively high, especially in 2003 and 2004. The average growth rate of energy consumption is approximately 7.88\% during 2000-2012. During 2003-2005, the energy consumption has experienced rapid growth and the growth rate is higher than $10 \%$. And there has been consistent growth from 2008-2012 in the energy consumption. However, the growth rate has maintained at a lower stage.

In terms of energy structure, coal, crude oil, natural gas and renewable energy accounted for $69.2 \%, 22.2 \%, 2.2 \%$ and $6.4 \%$ of total energy consumption in 2000 respectively, while the corresponding ratio is $68.0 \%, 19.0 \%, 4.4 \%$ and $8.6 \%$ in 2010 . The energy structure optimization in line with decrease of coal and increase of renewable energy consumption contributes to energy-economy sustainable development and helps protect environment.

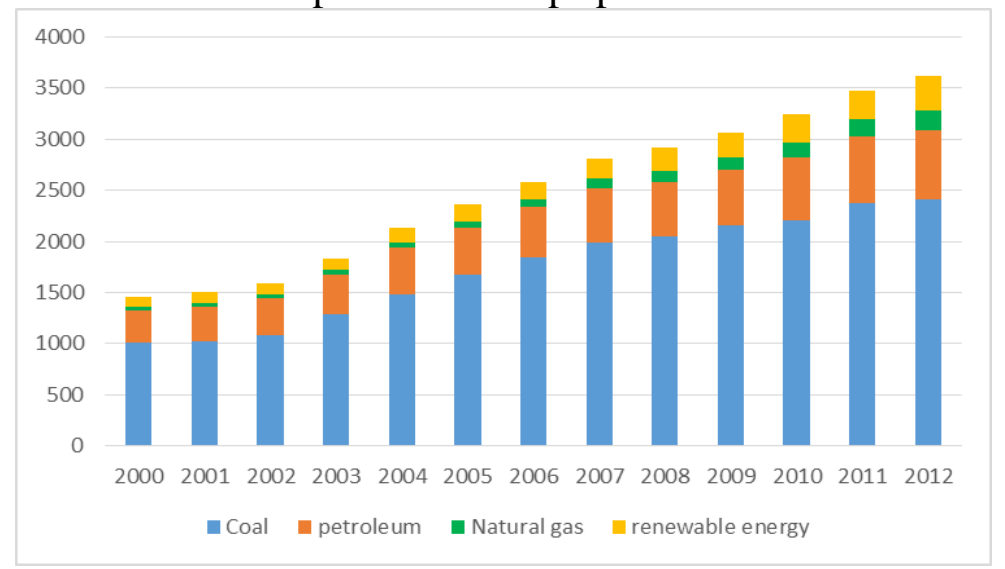

Fig.1: The total energy consumption and growth rate in China during 2000-2012 (Unit: Mtce) 
Unit Root Test. The purpose of Unit Root Test is to identify the existence of the unit root. Because the presence of unit root indicate the non-stationary time series. Furthermore, it will result in spurious regression. ADF test is the most common method of unit root test. The series of total energy consumption in China take natural logarithm to avoid heterosexuality. We apply the ADF test to investigate the existence of unit root in China's energy consumption. Energy consumption raises obviously from 2010 to 2012. The ADF value in its level is -2.596198, which is higher than the critical value of significance level $10 \%, 5 \%$ and $1 \%$ respectively. Thus it can not reject the existence of unit root of. According to unit root test, the series of China's energy consumption appear to contain a unit root in its level indeed. China's energy consumption is non-stationary time series. But the ADF value in 1st difference is -4.034017 , which is higher than the critical value of significance level $10 \%$ and $5 \%$ respectively. It is stationary in the 1st difference. The result shows that it is integrated at order one, namely I (1). The specific results are displayed in Table 1.

Table 1: ADF test

\begin{tabular}{llllll}
\hline \multirow{2}{*}{ Test critical value (\%) } & \multicolumn{2}{l}{ Unit root in level } & & $\begin{array}{l}\text { Unit root } \\
\text { difference }\end{array}$ & in 1 st \\
\cline { 2 - 3 } \cline { 5 - 6 } & T-Statistic & Prob. & & T-Statistic & Prob. $^{*}$ \\
& -2.596198 & 0 & & -4.034017 & 0 \\
\hline 1 & -5.1152 & & & -5.2735 & \\
5 & -3.927 & & -3.9948 & \\
10 & -3.4104 & & -3.4455 & \\
\hline
\end{tabular}

Model Identification. First-order differential operation will be conducted for the time series of energy consumption. The next step is to test auto-correlation and partial auto-correlation based on difference sequence. Fig. 2 shows the results of correlation analysis. According to auto-correlation coefficients and partial auto-correlation coefficients, we try to fit a random time series ARIMA $(1,1,0)$ model, ARMA $(1,1,0)$ including the intercept or trend, ARIMA $(1,1,1)$ model, ARIMA $(1,1,1)$ model including the intercept or trend. The auto-correlation coefficient and partial auto-correlation coefficient of $\triangle G D P$ are first-order truncation. Accordingly, the ARIMA $(1,1,1)$ model is constructed.

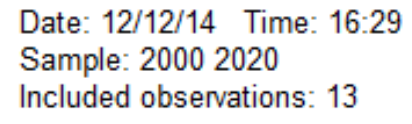

\begin{tabular}{|c|c|c|c|c|c|c|c|c|}
\hline \multicolumn{2}{|c|}{ Autocorrelation } & \multicolumn{2}{|c|}{ Partial Correlation } & \multicolumn{2}{|r|}{$\mathrm{AC}$} & PAC & Q-Stat & Prob \\
\hline 1 & & 1 & & 1 & 0.561 & 0.561 & 5.1149 & 0.024 \\
\hline 1 & 1 & 1 & 1 & 2 & 0.030 & -0.415 & 5.1310 & 0.077 \\
\hline 15 & 1 & 1 & 1 & 3 & -0.259 & -0.088 & 6.4370 & 0.092 \\
\hline 1 & 1 & & 1 & 4 & -0.334 & -0.140 & 8.8569 & 0.065 \\
\hline 15 & 1 & 1 & 1 & 5 & -0.372 & -0.266 & 12.224 & 0.032 \\
\hline $1 \square$ & 1 & 1 & 1 & 6 & -0.218 & 0.078 & 13.548 & 0.035 \\
\hline 1 & 1 & 1 & 1 & 7 & -0.050 & -0.148 & 13.630 & 0.058 \\
\hline 1 & 1 & $\square$ & 1 & 8 & -0.029 & -0.217 & 13.664 & 0.091 \\
\hline 1 & 1 & I & 1 & 9 & -0.049 & -0.070 & 13.782 & 0.130 \\
\hline 1 & 1 & 1 & 1 & 10 & 0.067 & 0.042 & 14.072 & 0.170 \\
\hline 1 & 1 & $1 \square$ & 1 & 11 & 0.083 & -0.209 & 14.747 & 0.194 \\
\hline
\end{tabular}

Fig.2: the 1st order differential auto-correlation and partial auto-correlation analysis

Then parameters in the model is in Fig.4. Based on the above analysis, we established the function of energy consumption as followed:

$$
\Delta E^{t}=177.55+0.29 \Delta E^{t-1}+\mu^{t}+0.48 \mu^{t-1}
$$




\begin{tabular}{ccccc}
\hline \hline Variable & Coefficient & Std. Error & t-Statistic & Prob. \\
\hline \hline C & 177.5463 & 39.59016 & 4.484608 & 0.0015 \\
AR(1) & 0.292348 & 0.456680 & 0.640160 & 0.5380 \\
MA(1) & 0.487275 & 0.530241 & 0.918969 & 0.3821 \\
\hline \hline
\end{tabular}

Fig.3: The results of parameter Estimation

Model Prediction. In the light of Eq. (2), we are able to forecast the energy consumption during 2000-2020. The real data and prediction data are shown in Table 2 and Table 3. Except for a few years, The absolute value of relative error rate is lower than $3 \%$, which indicates that the predicted data and the actual data is relatively close. The ARIMA $(1,1,1)$ model of total energy consumption is better overall fit. It has a high fitting precision and a certain stability. Therefore, the model in this paper has a higher fitting degree. It can be used to predict China's energy consumption in future. China's energy consumption is likely to increase by the end of 2020. The energy consumption is predicted to 4450Mtce in 2020. Moreover, the growth rate of China's energy consumption during 2013-2020 continues to decline. It is mainly because the pace of economic development slows down and energy efficiency improves in the future development.

Table 2: The comparison between real data and prediction data of China's energy consumption during 2008-2012

\begin{tabular}{lccccc}
\hline & 2008 & 2009 & 2010 & 2011 & 2012 \\
\hline Real data (Mtce) & 2914.48 & 3066.47 & 3249.39 & 3480.00 & 3617.00 \\
Prediction data (Mtce) & 2966.89 & 3146.21 & 3312.71 & 3467.30 & 3610.83 \\
Relative error (\%) & 1.80 & 2.60 & 1.95 & 0.37 & 0.17 \\
\hline
\end{tabular}

Table 3: Predictive value of China's energy consumption during 2013-2020

\begin{tabular}{lcccccccc}
\hline & 2013 & 2014 & 2015 & 2016 & 2017 & 2018 & 2019 & 2020 \\
\hline Prediction & 3744.09 & 3867.83 & 3982.72 & 4089.39 & 4188.43 & 4280.39 & 4365.72 & 4445.06 \\
Value (Mtce) & & & & & & & & \\
Growth rate & 3.51 & 3.30 & 2.97 & 2.68 & 2.42 & 2.20 & 1.99 & 1.82 \\
\hline
\end{tabular}

Comparison with the Target. China's energy long-term (2030, 2050) Development Strategy Research Report pointed out that China's total energy consumption in 2015 is suitable for controlling at 4000 Mtce. China Energy Bureau declared that China makes effort to control the total primary energy consumption at 4800Mtce million. Compared with the target, the predicted value is 3982.72 Mtce in 2015 and 4445.06 Mtce. it is very close to the target value in 2015 and less than the target value in 2020. That is to say, energy consumption at the current rate will meet the anticipated goals.

According to 18th National Congress of the Communist Party of China, the GDP will be more than double by 2020 . The energy intensity in 2020 can be calculate and the specific values is 0.028 tce/1000 yuan. It will drop by $65.80 \%$ compared with that in 2010 .

\section{Conclusion}

In this paper, the ARIMA $(1,1,1)$ model is established to forecast the energy consumption in the future. Through the model parameter estimation, it finds that the relative error for energy consumption prediction is small. Thus the model performs high prediction accuracy. It is used to forecast energy consumption during 2013-2020. The results indicate China's energy consumption will continue to increase by the end of 2020. Though the predicted energy consumption in 2015 and 2020 is lower than target value. Increasing energy consumption will result in energy shortage. Undoubtedly, it will impose a negative impact on economic growth. In order to balance economic 
growth and energy consumption, effective policies on energy-saving and energy efficiency improvement should be adopted. Full attention should be payed to economic restructuring and potential mining to reduce energy energy consumption. And effective policies also include vigorously promoting the industrial structure optimization, the transformation of economic growth and development the new industry with high technology content, added value, low energy consumption.

\section{References}

[1] L. Suganthi, Anand A. Samuelb. Energy models for demand forecasting-A review. Renewable and Sustainable Energy Reviews, 2012.

[2] Cheng, Zhou, and Chen Xiyang. Adaptive Combination Forecasting Model Based on Area Correlation Degree with Application to China's Energy Consumption. Journal of Applied Mathematics, 2014.

[3] Hor C.L. Watson S.J., Majithia S. Daily Load Forecasting and Maximum Demand Estimation using ARIMA and GARCH. Probabilistic Methods Applied to Power Systems. International Conference, 2006.

[4] Liu Yong, Wang Xuhui. China's energy consumption Forecasting based on ARIMA model. Economic Survey Section, 2007.

[5] China statistical yearbook. Beijing: China Statistics Press (1990-2010)

[6] Ruijun Xing, Liying Lin. based on ARIMA model for energy consumption Prediction. Statistics and Decision, 2008

[7] Aiqin Lin. Combined model for energy consumption prediction. China Population, Resources and Environment, 2010.

[8] Tiemei Gao. Econometric analysis and modeling. Tsinghua University Press, Beijing, 2006.

[9] Hsiao-Tien Pao, Chung-Ming Tsai. Modeling and forecasting the CO2 emissions, energy consumption, and economic growth in Brazil. Energy, 2011. 